\title{
Characterization of influenza A virus pseudotyped with the spike protein of porcine epidemic diarrhea virus
}

\author{
Asawin Wanitchang ${ }^{1} \cdot$ Janya Saenboonrueng ${ }^{1} \cdot$ Kanjana Srisutthisamphan $^{1} \cdot$ Anan Jongkaewwattana ${ }^{1} \mathbb{C}$
}

Received: 11 June 2018 / Accepted: 2 August 2018 / Published online: 22 August 2018

C) Springer-Verlag GmbH Austria, part of Springer Nature 2018

\begin{abstract}
The coronavirus spike protein and the influenza virus hemagglutinin are class I viral membrane fusion proteins. While the two proteins display strong structural conservation and the mechanisms underlying membrane fusion are similar, they share no sequence similarity. Whether they are functionally interchangeable is currently unknown. In this study, we constructed scIAV-S, a single-cycle influenza A virus pseudotyped with the spike protein of porcine epidemic diarrhea virus (PEDV), and demonstrated that this virus could infect cultured cells and trigger massive syncytium formation. Treatment with endocytosis inhibitors did not affect syncytium formation by infected cells. Moreover, the infectivity of scIAV-S was associated with the degree of cell adaptation of PEDV-S. Intriguingly, scIAV-S lacking functional neuraminidase (NA) exhibited substantially higher infectivity, suggesting a pivotal role of the sialic acid in the binding/entry of PEDV. Together, scIAV-S offers a robust platform for the investigation of the entry mechanism of PEDV or, possibly, of other coronaviruses.
\end{abstract}

\section{Introduction}

Porcine epidemic diarrhea virus (PEDV), a member of the genus Alphacoronavirus, is a pleomorphic enveloped virus that contains a large (approximately $28 \mathrm{~kb}$ ), positive-sense, single-stranded RNA genome. It is a causative agent of PED, a highly contagious gastrointestinal disease in pigs with mortality reaching $100 \%$ in suckling piglets. While PEDV replicates efficiently in porcine enterocytes in vivo, it grows poorly in cultured cells unless adapted through multiple passages [6]. Like those of other coronaviruses (CoVs), the PEDV spike protein (PEDV-S) is responsible for both receptor recognition and fusion between the virus and host cells $[10,15]$. Notably, the identity of the receptor used by PEDV is still a subject of controversy. While earlier studies have suggested that PEDV uses porcine aminopeptidase $\mathrm{N}$ (pAPN) as a cellular receptor $[9,12,22,24]$, more-recent

Handling Editor: William G Dundon.

Anan Jongkaewwattana

anan.jon@biotec.or.th

1 Virology and Cell Technology Laboratory, National Center for Genetic Engineering and Biotechnology (BIOTEC), Nation Science and Technology Development Agency (NSTDA), 113 Thailand Science Park, Phahonyothin Rd., Klong 1, Klong Luang, Pathum Thani 12120, Thailand studies have supported a contrary view based on several lines of evidence [17, 29].

CoV-S proteins, including PEDV-S, are members of the class I viral membrane fusion proteins, which also include hemagglutinin (HA) from influenza A virus (IAV) [3, 14]. Both CoV-S and HA fold into a metastable prefusion conformation and require proteolytic cleavage (priming) by host proteases to make them fusogenic. This cleavage produces a receptor-binding subunit (HA1 of influenza virus or S1 of $\mathrm{CoV}-\mathrm{S}$ ) and a membrane-fusion subunit (HA2 or S2), which remain associated through non-covalent interactions. While details of the prefusion structure of PEDV-S are lacking, recent studies of the spike proteins of the betacoronaviruses MHV and HKU1 have suggested that the overall prefusion structures of CoV-S and HA are similar [14] and that they might share a common mechanism of membrane fusion. However, a few fundamental differences between the two proteins should also be considered. While the fusion peptide of HA is located at the N-terminus of HA2, CoV-S has an internal fusion peptide located downstream of the $\mathrm{N}$-terminus of S2. Consequently, two proteolysis sites are usually required for the conformational change of $\mathrm{S} 2$ : one at the $\mathrm{S} 1 / \mathrm{S} 2$ junction and the other at the so-called S2' site located at the $\mathrm{N}$-terminus of the fusion peptide $[1,2]$. In addition, unlike HA, many CoV-S, proteins probably including that of PEDV, are not sufficiently primed by proteolysis during viral packaging release and thus require further 
proteolysis by extracellular proteases, cell-surface proteases, or lysosomal proteases [14]. Considering these common and unique properties of the two proteins, it would be interesting to investigate whether they could functionally replace each other in a virus infection context.

Recombinant retrovirus pseudotyped with PEDV-S has been generated and employed to investigate the PEDV entry mechanism [20, 33]. However, cells transduced by pseudotyped retrovirus usually display characteristics that are distinct from those of cells that are infected with PEDV. For example, cell-cell fusion is rarely observed in transduced cells. Notably, with the successful development of a system to construct single-cycle influenza A virus (scIAV) $[23,36]$, it is now possible to assess whether scIAV can be generated when PEDV-S is provided in trans instead of HA. The resulting pseudotyped virus (scIAV-S) will be beneficial especially for studying entry mechanism mediated by PEDVS. The present study was undertaken to test the hypothesis that PEDV-S can functionally replace HA to drive replication of IAV by constructing scIAV-S and examining its ability to infect PEDV-permissive cells. We also showed, using our pseudotyped virus system, that sialic acid is critical for mediating PEDV entry. In addition, we demonstrated that IAV carrying PEDV-S derived from cell-adapted and field-isolated strains exhibited distinct characteristics in infected cells, suggesting different modes of entry among PEDV strains.

\section{Materials and methods}

\section{Cells and viruses}

Human embryonic kidney (HEK) 293T, VeroE6-APN, and Madin-Darby canine kidney (MDCK) cells were maintained at $37{ }^{\circ} \mathrm{C}$ in Opti-MEM (Thermo Scientific) supplemented with $10 \%$ heat-inactivated fetal bovine serum (FBS) and $100 \mathrm{IU}$ of penicillin and $100 \mathrm{mg}$ of streptomycin per $\mathrm{ml}$ in humidified 5\% $\mathrm{CO}_{2}$ incubators. All scIAV-PEDV-S used in this study were generated in the HEK293T cells and stored at $-80{ }^{\circ} \mathrm{C}$ until use. Recombinant PEDV AVCT12 $_{12}$ was generated by reverse genetics and propagated in VeroE6-APN cells as described previously [8, 37]. Influenza A virus (A/ $\mathrm{PR} / 8 / 34$ ) was generated and titrated as described previously [34].

\section{Plasmid construction}

The full-length PEDV S derived from PEDV $\mathrm{AVCT12}_{\text {(Gen- }}$

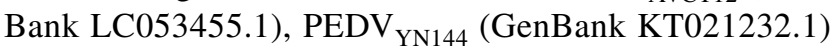
and $\mathrm{PEDV}_{\mathrm{G} 2}$ (field isolate) were codon-optimized for expression in mammalian cells and synthesized (Genscript and Synbio Tech). Subsequently, each construct was subcloned into the pCAGGS expression plasmid. To ensure optimal surface expression, ER retention signals at the C-terminal end were removed from all constructs.

The pHW-HA-mCherry and pHW- $\Delta \mathrm{M} 2$ plasmids were constructed as described previously [36]. To construct pHW$\Delta \mathrm{NA}, \mathrm{pHW} 2000$ encoding the NA gene of $\mathrm{A} / \mathrm{PR} / 8 / 34$ was subjected to site-directed mutagenesis to introduce two consecutive stop codons at amino acids 164 and 165. All plasmids were subjected to nucleotide sequencing to ensure that no unwanted mutations were inadvertently introduced.

\section{Recovery of ScIAV -S}

scIAV-S expressing the mCherry protein were rescued using a strategy similar to one described in a previous study [36]. Briefly, HEK293T cells in a six-well plate were transfected with $0.5 \mu \mathrm{g}$ each of pHW2000 plasmids encoding the seven segments (PB2, PB1, PA, NP, NA, M, and NS) from A/ $\mathrm{PR} / 8 / 34$ and pHW-HA-mCherry together with $2 \mu \mathrm{g}$ of the pCAGGS plasmid encoding each construct of PEDV-S, using Fugene HD (Promega). To construct scIAV-S lacking NA, pHW- $\triangle$ NA was used instead of pHW2000-NA. Likewise, scIAV-S lacking M2 was constructed by using pHW- $\Delta \mathrm{M} 2$ instead of pHW2000-M. At $72 \mathrm{~h}$ after transfection, cell supernatants were harvested and adsorbed directly onto VeroE6-APN cells for further analysis.

\section{Western blot assay}

Western blot assays were carried out according to the published procedure with some modifications [35]. Transfected cells were collected and lysed in $200 \mu \mathrm{l}$ of mammalian cell lysis buffer (50 mM Tris [pH 8.0], $5 \mathrm{mM}$ EDTA, $100 \mathrm{mM}$ $\mathrm{NaCl}, 1 \% \mathrm{NP}-40$ and protease inhibitor mixture) for $30 \mathrm{~min}$ on ice. After centrifugation at $10,000 \times g$ for $5 \mathrm{~min}$, lysates were separated on $10 \%$ SDS-PAGE gels and subsequently transferred to nitrocellulose membranes (Bio-Rad), followed by blocking with 5\% non-fat milk in TBS-T for $1 \mathrm{~h}$. Membranes were probed with one of the primary antibodies, including anti-PEDV-S mouse polyclonal antibody (a kind gift from Dr. Qigai $\mathrm{He}$ ), and anti- $\beta$-actin mouse monoclonal antibody clone C-4 (Santa Cruz Biotechnology) followed by goat anti-mouse antibodies conjugated to HRP (Biolegend). The signals were visualized with western blotting detection reagent (Bio-Rad).

\section{Immunofluorescence assay}

VeroE6-APN cells were grown on a Lab-Tek II chamber slide (Thermo Scientific) in Opti-MEM supplemented with $10 \% \mathrm{FBS}$ for $12 \mathrm{~h}$ at $37^{\circ} \mathrm{C}$. The adherent cells were inoculated with scIAV-S for $1 \mathrm{~h}$ at $37^{\circ} \mathrm{C}$. After three washes with PBS, cells were cultured in serum-free Opti-MEM 
in the presence of trypsin $(2 \mu \mathrm{g} / \mathrm{ml})$ for $24 \mathrm{~h}$. Cells were fixed in $80 \%$ chilled acetone for $10 \mathrm{~min}$ and blocked in $10 \%$ FBS/1\%BSA/PBS for $30 \mathrm{~min}$. The slides were subsequently incubated with mouse anti-influenza-A-virus NP antibodies (Clone 2C9; Southern Biotech) for $1 \mathrm{~h}$, washed three times with PBS, and incubated with FITC-conjugated anti-mouse $\mathrm{IgG}$ antibodies. After additional washes, the slides were mounted with Antifade Mounting Medium with DAPI (Vector Laboratories). Cells were examined using an Olympus IX51 fluorescence microscope.

\section{Statistical analysis}

Data were expressed as mean \pm SD. Statistical tests were performed using the Student $t$-test in GraphPad Prism 5.0 (GraphPad Software).

\section{Results}

\section{Recovery of ScIAV pseudotyped with PEDV $\mathrm{AVCT}_{12}$ spike (sclAV-S AVCT12 $_{\text {) }}$}

Recently, we established a reverse genetics system to construct scIAV in which the HA gene was replaced by the mCherry gene. The virus was recovered in HEK293T cells and subsequently propagated in MDCK cells stably expressing the HA protein [36]. Using a similar strategy, we attempted to rescue scIAV-S $\mathrm{AVCT12}_{\text {by transfecting }}$ HEK293T cells with plasmids necessary for IAV reverse genetics together with codon-optimized $\mathrm{S}_{\mathrm{AVCT} 12}$ as depicted in Fig. 1A. Notably, we did not add trypsin to transfected cells during virus rescue to avoid the detachment of HEK293T cells. Moreover, since cells expressing $S_{\text {AVCT12 }}$ underwent rapid cell-cell fusion, cells stably expressing $\mathrm{S}_{\mathrm{AVCT} 12}$ could not be obtained for the propagation of scIAV$\mathrm{S}_{\mathrm{AVCT12}}$. Alternatively, the trypsin-free viruses released from HEK293T cells were directly inoculated onto VeroE6-APN cells treated with trypsin $12 \mathrm{~h}$ after infection. At 24 after infection, mCherry expression was assessed by fluorescence microscopy. Unexpectedly, not only did we observe a strong mCherry signal, but we also detected extensive syncytia in

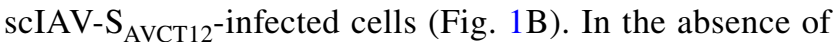
trypsin treatment, we could still detect a few cells with an mCherry signal but no syncytium formation (Fig. 1B), suggesting that scIAV-S ${ }_{\text {AVCT12 }}$ might be able to enter cells but trypsin is essential for induction of cell-cell fusion. It should also be noted that control supernatants from HEK293T cells transfected with pCAGGS expressing $\mathrm{S}_{\mathrm{AVCT} 12}$ alone did not yield detectable syncytium formation in VeroE6-APN cells, suggesting that the detected syncytia were not due to PEDV$\mathrm{S}$ expressed from residual plasmids (data not shown).
To characterize scIAV-S $\mathrm{AVCT12}_{2}$, we initially tried to purify rescued viruses from the supernatants and perform western blot analysis to detect co-expression of PEDV-S and IAV nucleoprotein (ANP). Unfortunately, despite several attempts, we could not obtain sufficient purified virus for the assay (data not shown). Alternatively, we performed immunofluorescence staining to examine the expression of ANP in the

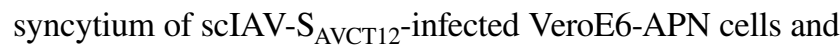
detected strong expression of ANP (Fig. 1C). To further test whether the mCherry expression in infected cells required active influenza A virus polymerase activity, we attempted to construct scIAV-S $\mathrm{S}_{\mathrm{AVCT} 12}$ by omitting the plasmid encoding the PB2 polymerase and examined its infectivity in VeroE6APN cells. As expected, in the absence of PB2, neither an mCherry signal nor syncytium formation could be detected in infected cells (Fig. 1D). These results suggest that scIAV can be pseudotyped with PEDV-S and that the pseudovirus can efficiently infect PEDV-permissive cells. It is also notable that the massive syncytium formation in scIAV-infected cells was unexpected, and this observation has never been reported elsewhere. This points to the possibility that scIAV-S might enter cells by a mechanism similar to that of PEDV.

\section{Abrogation of $\mathrm{M} 2$ ion channel activity does not affect ScIAV-S AVCT12 $_{\text {infectivity }}$}

Given that scIAV-S $\mathrm{S}_{\mathrm{AVCT} 12}$-infected cells formed large syncytia in the presence of trypsin (Fig. 1B) and that $\mathrm{S}_{\mathrm{AVCT} 12}$ was not synthesized de novo in scIAV-S $\mathrm{S}_{\mathrm{AVCT} 12}$-infected cells, we speculated that the observed syncytium formation might have been mediated by $\mathrm{S}_{\mathrm{AVCT} 12}$ remaining at the cell surface following internalization of scIAV-S $\mathrm{S}_{\mathrm{AVCT} 12}$. In other words, replacing HA with $\mathrm{S}_{\mathrm{AVCT} 12}$ might have switched the mode of cell entry of scIAV-S $\mathrm{S}_{\mathrm{AVCT} 12}$ from endocytosis to direct fusion at the plasma membrane. Moreover, since the M2 ion channel is essential for the uncoating of IAV in host cell endosomes, entry of

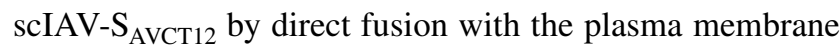
would bypass viral-endosomal fusion, rendering the proton channel function of M2 unnecessary. To test this hypothesis, we constructed scIAV-S $\mathrm{SVCT}_{\mathrm{A} 12}$ lacking a functional M2 ion channel (scIAV $\Delta \mathrm{M} 2-\mathrm{S}_{\mathrm{AVCT} 12}$ ) and assessed its infectivity in VeroE6-APN cells. As shown in Fig. 2, we detected undisturbed mCherry expression with large syncytium formation in scIAV $\Delta \mathrm{M} 2-\mathrm{S}_{\mathrm{AVCT} 12}$-infected cells when infected cells were treated with trypsin. Notably, we have shown previously that scIAV $\Delta \mathrm{M} 2$ carrying IAV HA showed no mCherry signal in MDCK cells [36]. 
Fig. 1 Construction and

infectivity of scIAV-S $\mathrm{AVCT12}_{\text {. A. }}$. Schematic representation of the IAV reverse genetics platform used to generate scIAV-S $\mathrm{S}_{\mathrm{AVCT} 12}$. B. scIAV-S $\mathrm{S}_{\mathrm{AVCT} 12}$ (undiluted) was adsorbed onto VeroE6-APN cells for $1 \mathrm{~h}$. The infected cells were further incubated in the absence or presence of trypsin $(2 \mu \mathrm{g} / \mathrm{ml})$ at $37^{\circ} \mathrm{C}$ for $24 \mathrm{~h}$. The expression of mCherry and nuclear staining (using Hoechst 33342 fluorescent stain) were examined using a fluorescence microscope. Scale bars, $20 \mu \mathrm{m}$ and $100 \mu \mathrm{m}$ (for no trypsin treatment) C. VeroE6-APN cells on chamber slides were infected with scIAV-S $\mathrm{S}_{\mathrm{AVCT} 12}$ (undiluted). At $24 \mathrm{~h}$ after infection, infected cells were fixed and subjected to immunofluorescence staining for ANP. Cells were also stained with DAPI to show nuclei. D. scIAV-S ${ }_{\mathrm{AVCT} 12}$ was constructed in the presence or absence of pHW2000 encoding PB2. The resulting viruses (undiluted) were adsorbed onto VeroE6APN cells for $1 \mathrm{~h}$. Infected cells were further incubated in the presence of trypsin $(2 \mu \mathrm{g} / \mathrm{ml})$ at $37^{\circ} \mathrm{C}$ for $24 \mathrm{~h}$. The expression of mCherry and syncytium formation were examined by fluorescence microscopy. Scale bars, $20 \mu \mathrm{m}$

A $A / P R / 8 / 34$ (H1N1)

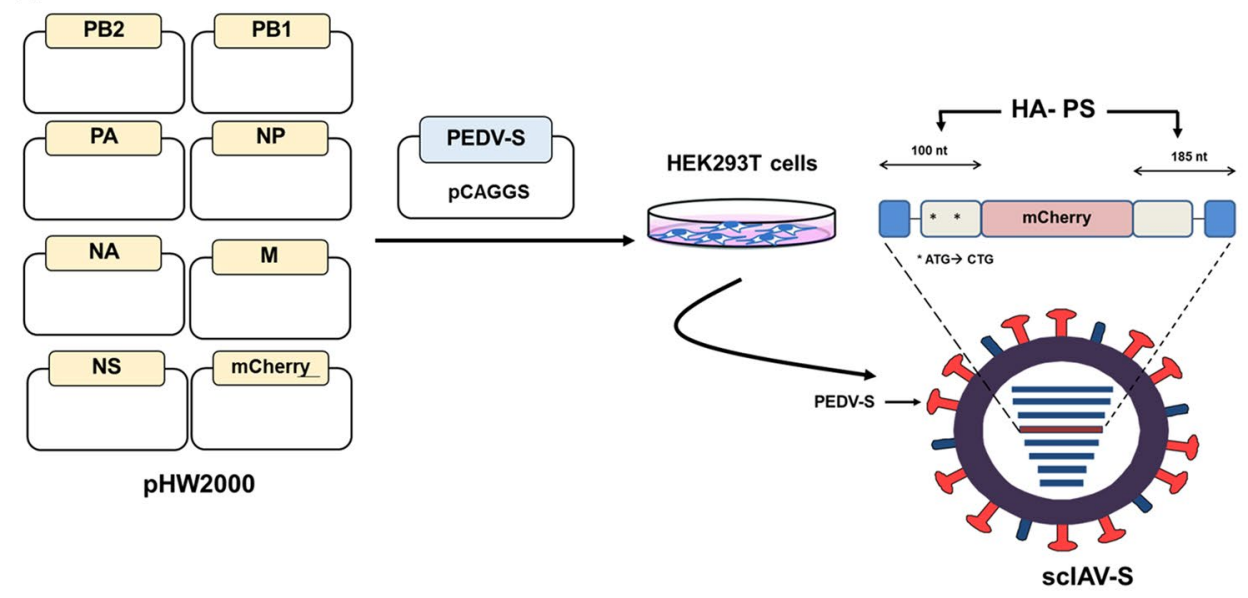

B

Bright field

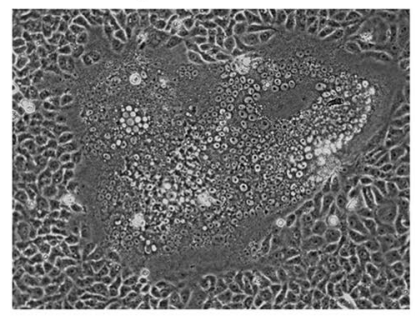

No trypsin treatment

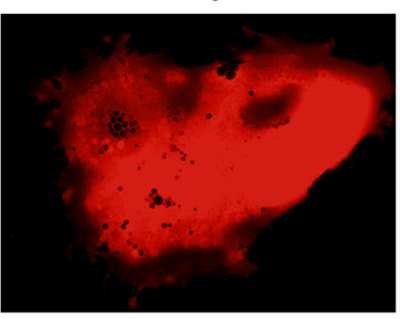

C

C ANP
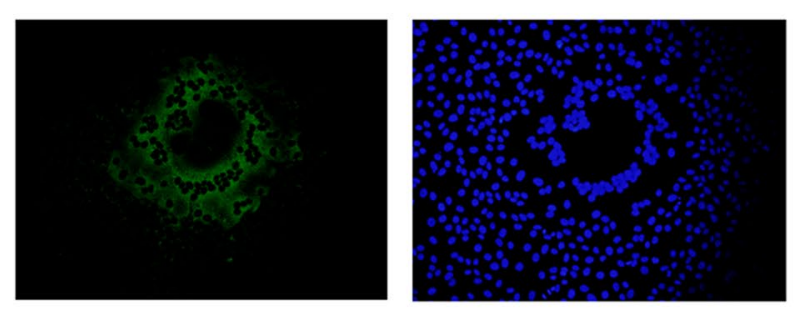

Wt

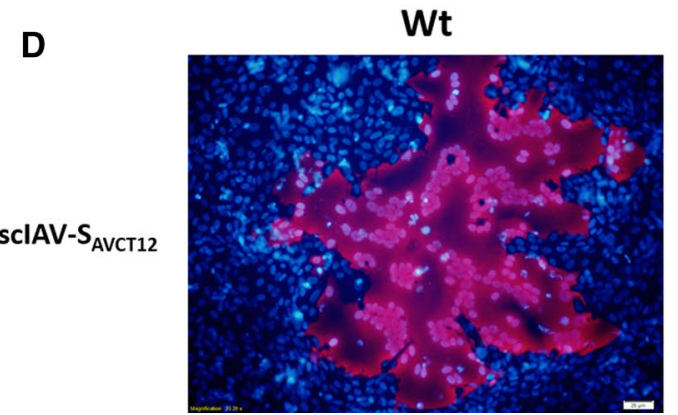

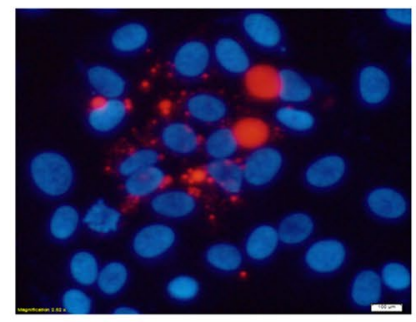

\section{Merged with Hoechst}
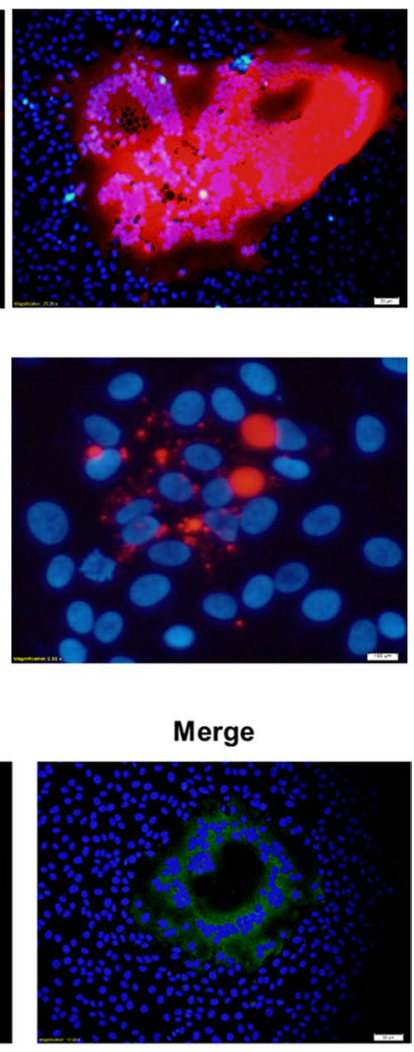

$\triangle \mathrm{PB} 2$

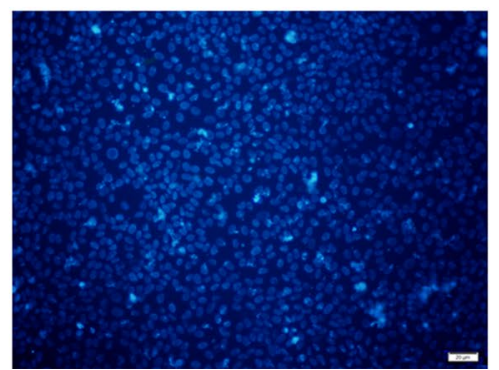



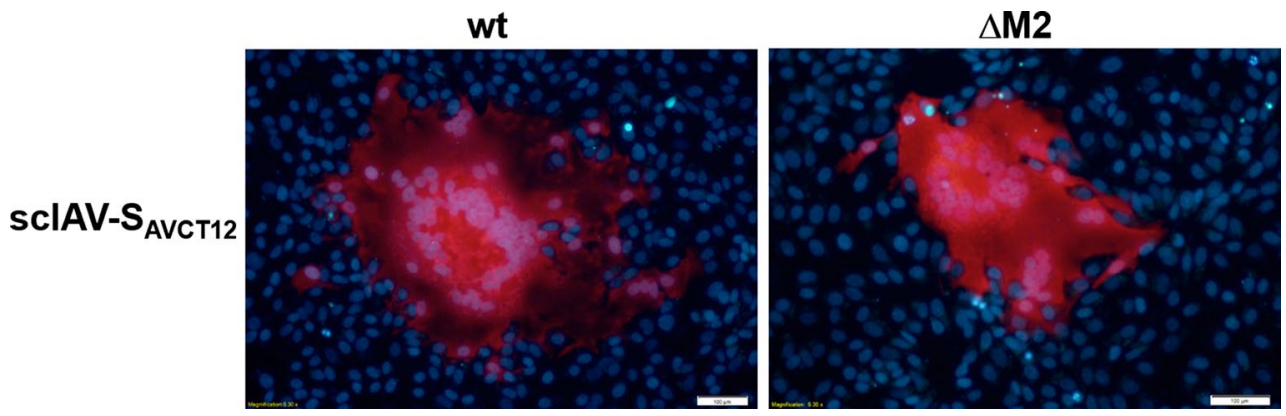

Fig. 2 M2 is not required for scIAV-S $\mathrm{S}_{\mathrm{AVCT} 12}$ infection. scIAV-S $\mathrm{S}_{\mathrm{AVCT} 12}$ was constructed using the pHW2000 plasmid encoding $M$ with a disrupted M2 ORF. The resulting virus was inoculated onto VeroE6APN cells for $1 \mathrm{~h}$. Infected cells were further incubated for $24 \mathrm{~h}$ in the presence of trypsin $(2 \mu \mathrm{g} / \mathrm{ml})$ before being examined under a fluorescence microscope for mCherry expression and syncytium formation. Scale bars, $100 \mu \mathrm{m}$

\section{Infectivity of scIAV-S depends on the degree of cell adaptation of the spike protein}

Several studies have shown that naturally isolated PEDV strains, mostly in the G2 genogroup, replicate poorly in Vero cells [25]. Accumulated mutations in the $S$ gene are strongly associated with cell adaptation [11, 18, 31]. It was therefore of interest to assess the infectivity of scIAV pseudotyped with $S$ derived from PEDV with various degrees of cell adaptation. $\mathrm{S}_{\mathrm{AVCT} 12}$ is closely related to the classical PEDV strain SM98-1, which is considered a highly cell-adapted strain. In addition, a variant (YN144) derived from a non-adapted strain after the $144^{\text {th }}$ passage in Vero cells [5] is deemed to be an intermediately adapted strain. We therefore synthesized a codonoptimized $\mathrm{S}$ gene $\left(\mathrm{S}_{\mathrm{G} 2}\right)$ based on a PEDV isolated from intestinal tissues of a piglet experiencing severe watery diarrhea during the outbreak in Thailand in 2014. BLAST analysis of the $\mathrm{S}_{\mathrm{G} 2}$ protein revealed that the amino acid sequence of our protein was $99.12 \%$ identical to that of CH/GXNN/2012 (GenBank AFO42860.1). Moreover, a codon-optimized S gene of strain YN144 was synthesized. As mentioned previously, both synthetic constructs were designed to exclude C-terminal ER retention motifs (Yxx $\Phi$ and $\mathrm{KxHxx}$ ). When plasmids expressing each PEDV-S were used to transfect VeroE6-APN cells in the presence of trypsin, only those expressing $\mathrm{S}_{\mathrm{AVCT} 12}$ and $\mathrm{S}_{\mathrm{YN} 144}$ displayed massive syncytium formation (Fig. 4A). The failure to form syncytia in PEDV-S $\mathrm{G}_{2}$-transfected cells was not due to defects in protein expression in transfected cells (Fig. 4A). We next attempted to construct scIAV pseudotyped with $\mathrm{S}_{\mathrm{G} 2}$ (scIAV- $\mathrm{S}_{\mathrm{G} 2}$ ) and with $\mathrm{S}_{\mathrm{YN} 144}$ (scIAV$S_{\text {YN144 }}$ ) and evaluated their infectivity in VeroE6-APN cells. As a control, scIAV-S AVCT12 $_{\text {was also generated and }}$ examined. While abundant mCherry-expressing cells and large syncytia were detected in the cell cultures infected a mechanism that is independent of the clathrin-mediated endocytosis pathway. 

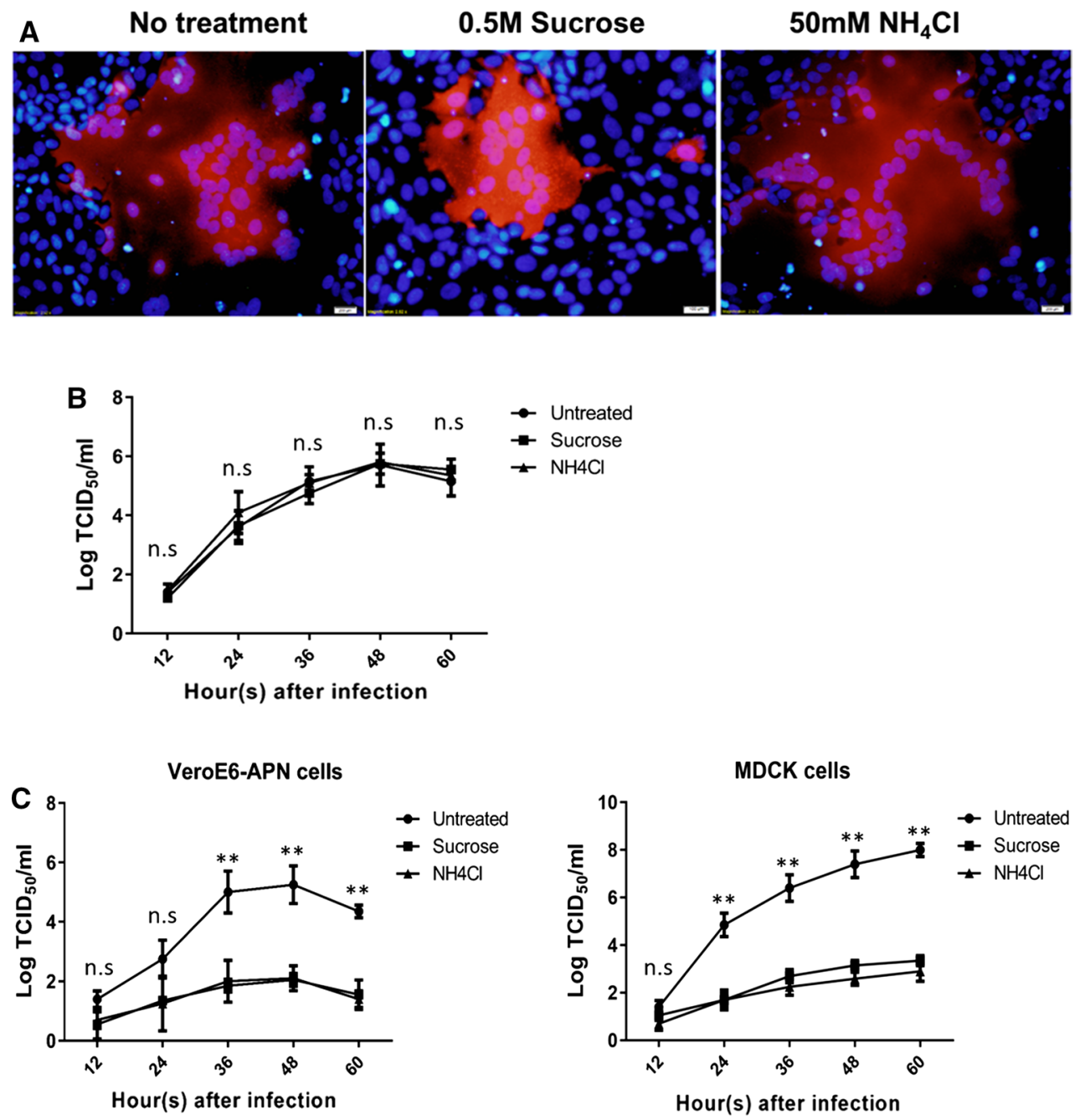

Fig. 3 Effect of hypertonic sucrose or $\mathrm{NH}_{4} \mathrm{Cl}$ treatment on entry of scIAV-S $\mathrm{AVCT12}_{12}$ and recombinant PEDV. A. VeroE6-APN cells were pretreated with $0.5 \mathrm{M}$ sucrose for $1 \mathrm{~h}$ or $50 \mathrm{mM} \mathrm{NH}_{4} \mathrm{Cl}$ for $2 \mathrm{~h}$ before being infected with scIAV-S $\mathrm{AVCT12}_{12}$. Untreated cells were used as a control. At $24 \mathrm{hpi}$, infected cells were examined for syncytium formation and mCherry expression under a fluorescence microscope. Scale bars, $100 \mu \mathrm{m}$. B. VeroE6-APN cells were mock treated or treated with $0.5 \mathrm{M}$ sucrose or $50 \mathrm{mM} \mathrm{NH}_{4} \mathrm{Cl}$ prior to infection with $\mathrm{PEDV}_{\mathrm{AVCT} 12}$ $(\mathrm{MOI}=0.1)$. Infected cells were maintained in Opti-MEM supplemented with trypsin $(2 \mu \mathrm{g} / \mathrm{ml})$. At the indicated time points after infection, supernatants were collected and evaluated for $\mathrm{TCID}_{50}$ in

with scIAV-S $\mathrm{S}_{\mathrm{AVCT} 12}$ and scIAV-S $\mathrm{S}_{\mathrm{YN} 144}$, those infected with scIAV-S $\mathrm{S}_{\mathrm{G} 2}$ displayed considerably fewer positive cells with punctate expression of mCherry and no apparent syncytium formation (Fig. 4B). The absence of syncytia in scIAV- $\mathrm{S}_{\mathrm{G} 2}$-infected cells may be due to an intrinsic property of PEDV-S $\mathrm{G}_{2}$, as cells transfected with pCAGGS$\mathrm{S}_{\mathrm{G} 2}$ also displayed less syncytium formation than those transfected with pCAGGS-S $\mathrm{S}_{\mathrm{AVCT} 12}$ and $-\mathrm{S}_{\mathrm{YN} 144}$ (Fig. 4A). Taken together, these results suggest that PEDV strains

VeroE6-APN cells. The mean \pm SD from three independent experiments is presented. n.s., not statistically significant. C. VeroE6-APN or MDCK cells were mock-treated or treated with $0.5 \mathrm{M}$ sucrose or $50 \mathrm{mM} \mathrm{NH}_{4} \mathrm{Cl}$ prior to infection with influenza A virus (A/PR/8/34; MOI $=0.01)$. Infected cells were maintained in Opti-MEM supplemented with TPCK-trypsin $(1 \mu \mathrm{g} / \mathrm{ml})$. At the indicated time points after infection, supernatants were collected and the amount of infectious virus $\left(\mathrm{TCID}_{50}\right)$ was determined in MDCK cells. The mean $\pm \mathrm{SD}$ from three independent experiments is presented. n.s., not statistically significant; $* *, p<0.01$

with different degrees of adaptation might utilize distinct pathways for internalization into host cells.

\section{Neuraminidase (NA) hinders the infectivity of SCIAV-S}

Several lines of evidence have indicated that PEDV utilizes sialic acid, possibly Neu5Ac, as a co-receptor for attachment and entry [13, 19]. Moreover, pretreatment of host cells with recombinant NA has been shown to substantially 
Fig. 4 Infectivity of scIAV bearing PEDV-S derived from viruses with different levels of cell adaptation. A. VeroE6APN cells were transfected with pCAGGS encoding $\mathrm{S}_{\mathrm{AVCT} 12}, \mathrm{~S}_{\mathrm{YN} 144}$ or $\mathrm{S}_{\mathrm{G} 2}(2 \mu \mathrm{g}$ each). Transfected cells were maintained in Opti-MEM supplemented with trypsin (2 $\mu \mathrm{g} / \mathrm{ml})$. Transfected cells were examined $24 \mathrm{~h}$ later for syncytium formation. Arrows denote syncytia. Likewise, HEK293T cells were transfected with pCAGGS encoding PEDV-S using the same conditions. At $48 \mathrm{~h}$ after transfection, cells were harvested, lysed and subjected to western blot analysis using anti-PEDV-S1 antibodies. Beta actin was used as a loading control. B. scIAV psedotyped with $\mathrm{S}_{\mathrm{AVCT} 12}, \mathrm{~S}_{\mathrm{YN} 144}$ or $\mathrm{S}_{\mathrm{G} 2}$ was constructed, and its infectivity in VeroE6-APN cells was evaluated. At $24 \mathrm{hpi}$, infected cells were examined for syncytium formation and mCherry expression under a fluorescent microscope. Scale bars, $50 \mu \mathrm{m}$ $\left(20 \mu \mathrm{m}\right.$ for scIAV-S $\mathrm{S}_{\mathrm{G} 2}$-infected cells)
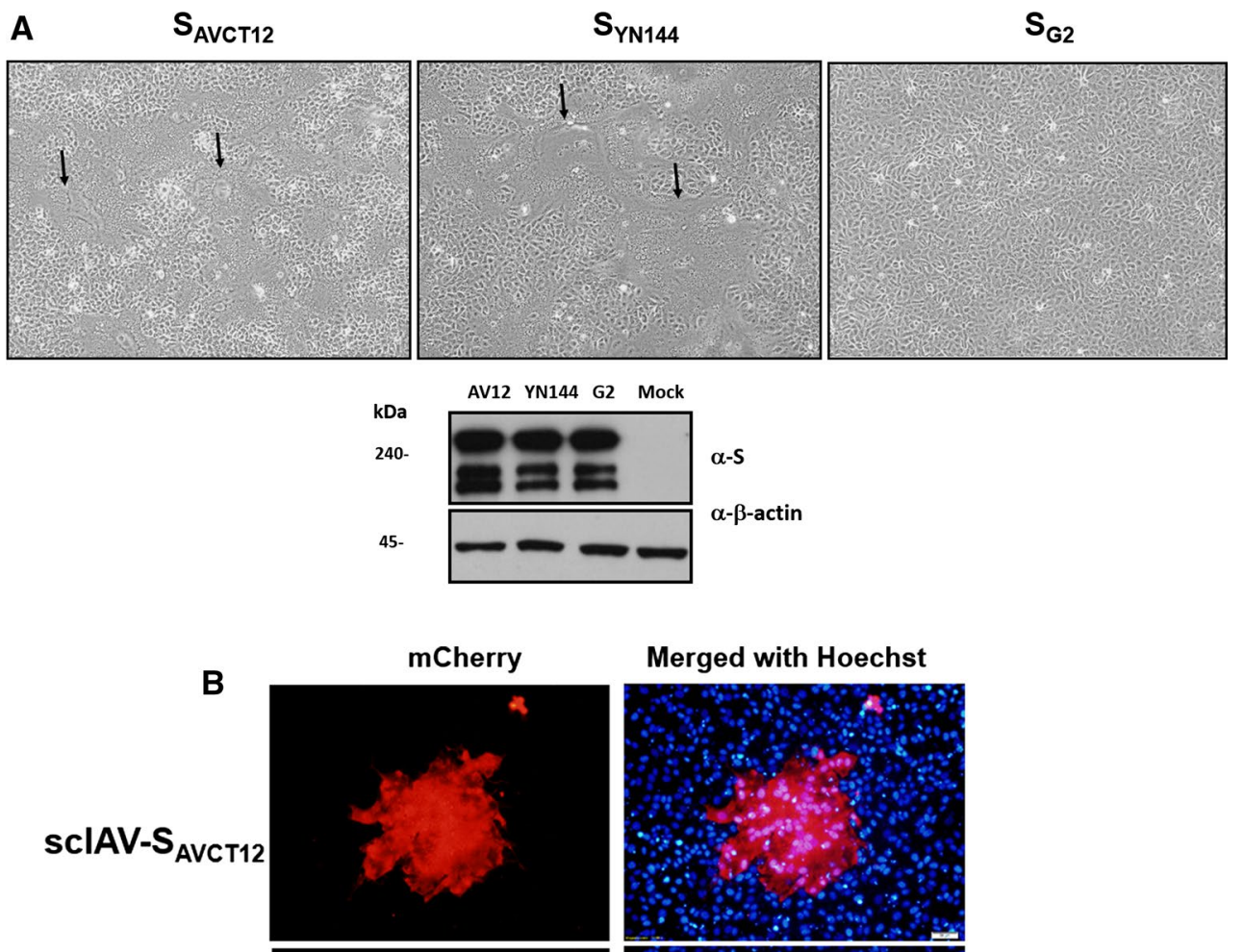

Merged with Hoechst
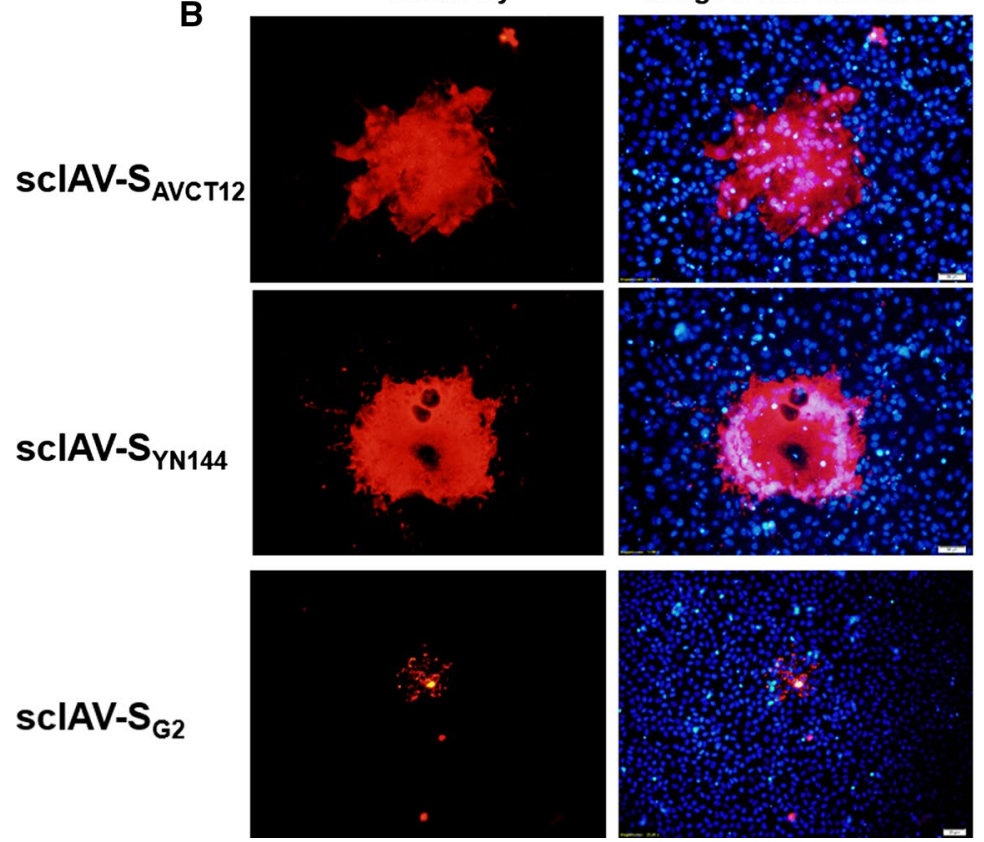

reduce PEDV infectivity, and the N-terminal domain (NTD) of PEDV-S (residues 1-249) has been proposed to be critical for sialic acid binding [9]. Unlike other previously described pseudotyped viruses, scIAV-S, produced in this study, contains active NA on its particles. It is therefore ideal for investigating the role of NA in infection by scIAV-S. Since scIAV$S$ does not require NA for release, we were able to construct an scIAV-S variant bearing a defective NA by introducing two stop codons in the NA gene $(\Delta \mathrm{NA})$. Of note, the NA deletion did not compromise the packaging signal of the NA gene, and therefore, packaging of the NA segment should be undisturbed. As expected, in the absence of NA, both scIAV$\mathrm{S}_{\mathrm{AVCT} 12}$ and $-_{\mathrm{YN} 144}$ could infect VeroE6-APN cells with substantially higher infectivity (Fig. 5A). While scIAV-S $\mathrm{S}_{\mathrm{G} 2}$ with $\triangle$ NA did not trigger syncytium formation in infected cells, we observed significantly more cells displaying an mCherry signal when compared to cells infected with the virus with intact NA (Fig. 5B). Taken together, these results indicate that the NA activity of scIAV-S might hinder the attachment of the PEDV-S on the scIAV-S particles with sialic acid on the cell surface.

\section{Discussion}

Constraints associated with in vitro culture of natural isolates of PEDV have considerably hindered the study of their replication, and, consequently, they have not yet been well characterized. In particular, the mechanism underlying how PEDV enters a host cell is not well understood. We demonstrate in this study that IAV particles could be efficiently pseudotyped with PEDV-S, and such pseudoviruses could 
Fig. 5 Effect of neuraminidase on the infectivity of scIAV-S. A. scIAV pseudotyped with $\mathrm{S}_{\mathrm{AVCT} 12}$ or $\mathrm{S}_{\mathrm{YN} 144}$ in the presence or absence of a functional NA gene in segment 6 was constructed. Syncytium formation was assessed in VeroE6APN cells (in a six-well plate) infected with each scIAV-S construct. The mean + SD from three independent experiments is presented. **, $p<0.01$. B scIAV pseudotyped with $\mathrm{S}_{\mathrm{G} 2}$ in the presence or absence of a functional NA gene in segment 6 was constructed. The expression of mCherry was assessed in infected VeroE6-APN cells at 24 hpi. Scale bars, $20 \mu \mathrm{m}$

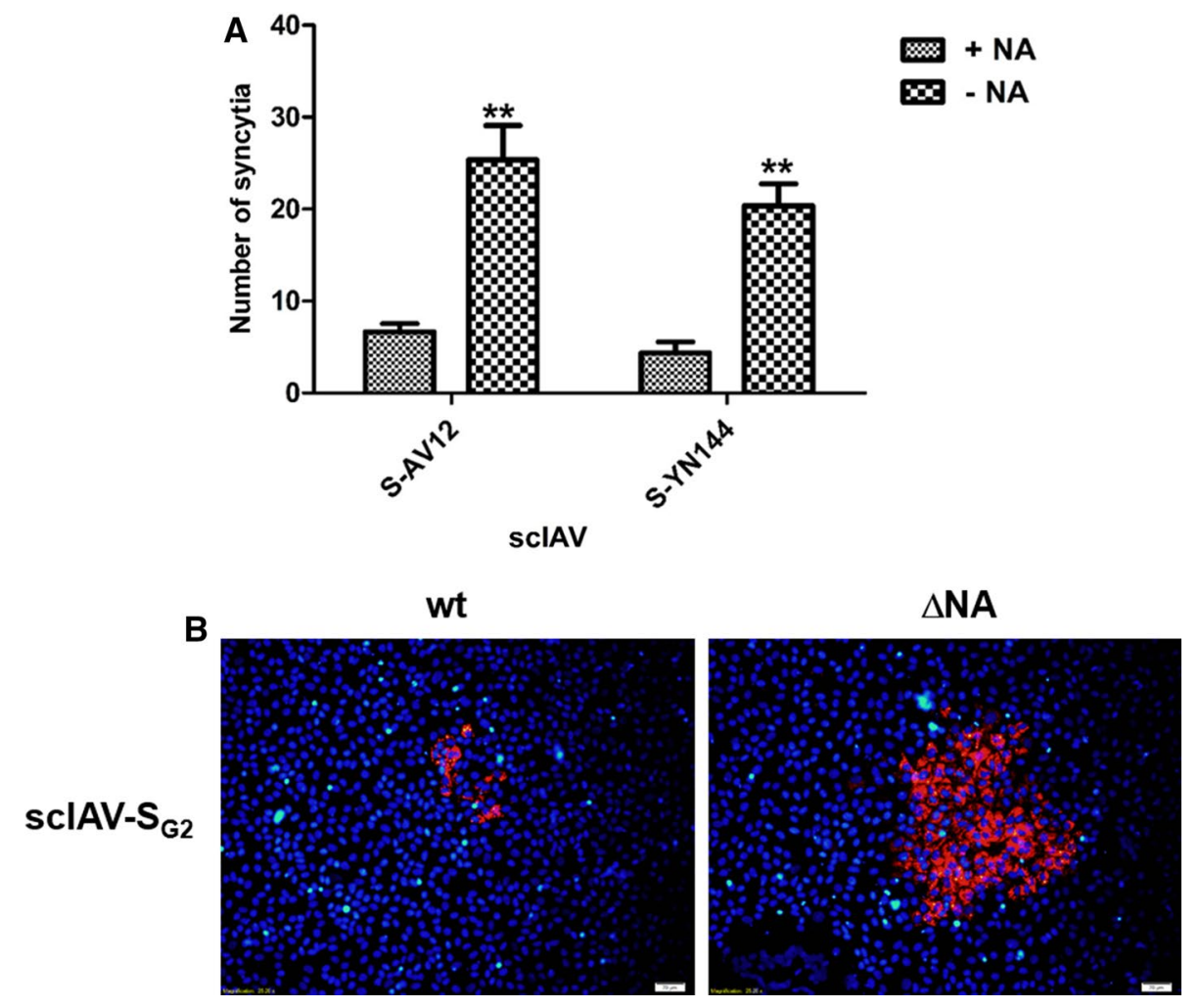

be employed to investigate the receptor binding and cellular entry of PEDV. Using a strategy similar to the one used with our previously described scIAV system [36], we successfully rescued scIAV carrying the mCherry gene in HEK293T cells co-expressing PEDV-S derived from various strains and demonstrated that scIAV-S $\mathrm{AVCT} 12$ could infect VeroE6-APN cells and produce a strong mCherry signal. Unfortunately, due to the high fusogenicity of $\mathrm{S}_{\mathrm{AVCT} 12}$, we could not generate cells stably expressing the protein as a platform to propagate scIAV-S $\mathrm{AVCT}_{22}$ in the current study. However, $\mathrm{S}_{\mathrm{AVCT} 12}$ is now being modified to be less fusogenic without compromising its receptor-binding function.

One of the most intriguing findings is our observation

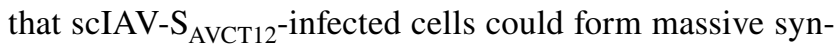
cytia similar to those produced by PEDV-infected cells. Since $\mathrm{S}_{\mathrm{AVCT} 12}$ is not synthesized de novo in scIAV-S $\mathrm{S}_{\mathrm{AVCT} 12^{-}}$ infected cells, the syncytium formation observed at neutral $\mathrm{pH}$ is likely to be due to interaction between the receptor and $\mathrm{S}_{\mathrm{AVCT} 12}$ remaining on the cell surface after internalization of the virus. Our data thus underscore the likelihood that scIAV-S $\mathrm{AVCT12}_{2}$ can enter cells by direct fusion at the plasma membrane. It is worth noting that some studies have shown that treatment with trypsin allows some CoVs to bypass the endosome-dependent pathway for entry [1, $21,30]$. However, scIAV-S $\mathrm{AVCT}_{12}$ was recovered in the absence of trypsin, and virus inoculation was carried out using trypsin-free viruses. Notably, unless trypsin was added at $12 \mathrm{hpi}$, we could not detect any syncytium formation.
Indeed, syncytium formation was entirely unexpected, as PEDV has been reported to enter cells by clathrin-mediated endocytosis even in the presence of large amounts of trypsin during infection [26]. We also showed that scIAV-S AVCT12 $_{\text {. }}$ lacking the M2 proton channel could infect VeroE6-APN cells and trigger formation of large syncytia similar to those observed in ScIAV-S $\mathrm{AVCT12}$-infected cells. In contrast to our previous report that ScIAV-HA requires functional $\mathrm{M} 2$ for infectivity [36], our findings here suggest that scIAV-S $\mathrm{AVCT12}_{\text {A }}$ can undergo replication without the need for M2-mediated internal virion acidification in the endosome. That PEDV can enter permissive cells by both direct fusion with the plasma membrane and endocytosis is not surprising as similar phenomena have also been observed with other CoVs [7, 30, 32].

In this study, we also speculated that differences in the $\mathrm{S}$ proteins of different PEDV strains might play a role in determining the mode of entry. In line with our hypothesis, Park and colleagues have recently demonstrated that the cleavage of the $\mathrm{S}$ protein during viral assembly appears to affect the mode of MERS-CoV entry. If $S$ is cleaved at the S1/S2 junction during biogenesis by host proteases such as furin or proprotein convertases in producer cells, the released virions are primed and able to enter target cells by fusing at the plasma membrane. Alternatively, virions released with uncleaved $\mathrm{S}$ need to be processed further by endocytic proteases in the target cell and therefore require endocytosis for entry [27]. Although PEDV-S, like other alphacoronaviruses, is 
believed to be present in an uncleaved form [15], data showing the cleavage of $\mathrm{S}$ in different PEDV strains are lacking. $S$ proteins of cell-adapted PEDV such as PEDV AVCT12 $_{\text {or }}$ $\mathrm{PEDV}_{\mathrm{YN} 144}$ may be cleaved during morphogenesis of the virion, whereas those of $\mathrm{PEDV}_{\mathrm{G} 2}$ may require further processing in the target cells. Further analysis of cleavage of the $\mathrm{S}$ protein in cell-adapted and field-isolated strains will be critical for better understanding of the mechanisms underlying PEDV entry.

Another important point worth mentioning is that we showed, using scIAV-S, that sialic acid moieties located on the cell surface plays a role in PEDV attachment. Although it has been demonstrated that the NTD of PEDV-S could bind to sialic acid, most data of the available have been based on assays using recombinant S proteins [13]. Because the construction of recombinant PEDV bearing specific mutations in the $\mathrm{S}$ gene can be technically challenging, the available data concerning the binding of PEDV S to sialic-acid-containing glycoproteins in the context of infection have so far been limited to the use of natural isolates such as the PEDVUU strain [15]. With a well-established IAV reverse genetics system, scIAV-S serves as a robust platform to assess binding of the $\mathrm{S}$ protein to sialic acid in the context of infection. Furthermore, since many CoVs, such as MERS-CoV [16], and transmissible gastroenteritis coronavirus (TGEV) [28], have been reported to bind to sialic acids, it is also possible to construct scIAV pseudotyped with $\mathrm{CoV}-\mathrm{S}$ of interest to investigate this interaction in more detail.

It is noteworthy that cells infected with scIAV-S display massive cell-cell fusion that is very similar to what is observed in cells infected with PEDV but does not occur in cells transduced with other pseudoviruses. Moreover, we showed here that PEDV-S derived from a field isolate, despite its poor growth in vitro, can be cloned into the expression vector and subsequently used to construct scIAV-S for further investigation. scIAV thus serves as a new platform not only for investigating the mechanism by which PEDV-S mediates cell entry but also to gain insights into the entry mechanisms utilized by cell-adapted and naturally isolated PEDV.

Acknowledgements We thank Dr. Qigai He for the kind gift of the anti-PEDV-S antibodies.

Funding This study was funded by National Center for Genetic Engineering and Biotechnology (BIOTEC)'s Fellow Grant, National Science and Technology Development Agency (P-15-51261) and Giga Impact Initiative Grant (P16-51674).

\section{Compliance with ethical standards}

Conflict of interest The authors have no financial conflicts of interest.

Human and animal rights statement Neither animal nor human testing was involved in this study.

\section{References}

1. Belouzard S, Chu VC, Whittaker GR (2009) Activation of the SARS coronavirus spike protein via sequential proteolytic cleavage at two distinct sites. Proc Natl Acad Sci USA 106:5871-5876

2. Belouzard S, Millet JK, Licitra BN, Whittaker GR (2012) Mechanisms of coronavirus cell entry mediated by the viral spike protein. Viruses 4:1011-1033

3. Bosch BJ, van der Zee R, de Haan CA, Rottier PJ (2003) The coronavirus spike protein is a class I virus fusion protein: structural and functional characterization of the fusion core complex. J Virol 77:8801-8811

4. Carpentier JL, Sawano F, Geiger D, Gorden P, Perrelet A, Orci L (1989) Potassium depletion and hypertonic medium reduce "noncoated" and clathrin-coated pit formation, as well as endocytosis through these two gates. J Cell Physiol 138:519-526

5. Chen F, Zhu Y, Wu M, Ku X, Ye S, Li Z, Guo X, He Q (2015) Comparative genomic analysis of classical and variant virulent parental/attenuated strains of porcine epidemic diarrhea virus. Viruses 7:5525-5538

6. Chen Q, Li G, Stasko J, Thomas JT, Stensland WR, Pillatzki AE, Gauger PC, Schwartz KJ, Madson D, Yoon KJ, Stevenson GW, Burrough ER, Harmon KM, Main RG, Zhang J (2014) Isolation and characterization of porcine epidemic diarrhea viruses associated with the 2013 disease outbreak among swine in the United States. J Clin Microbiol 52:234-243

7. Huang IC, Bosch BJ, Li F, Li W, Lee KH, Ghiran S, Vasilieva N, Dermody TS, Harrison SC, Dormitzer PR, Farzan M, Rottier PJ, Choe H (2006) SARS coronavirus, but not human coronavirus NL63, utilizes cathepsin L to infect ACE2-expressing cells. J Biol Chem 281:3198-3203

8. Jengarn J, Wongthida P, Wanasen N, Frantz PN, Wanitchang A, Jongkaewwattana A (2015) Genetic manipulation of porcine epidemic diarrhoea virus recovered from a full-length infectious cDNA clone. J Gen Virol 96:2206-2218

9. Kamau AN, Park JE, Park ES, Yu JE, Rho J, Shin HJ (2017) Porcine amino peptidase $\mathrm{N}$ domain VII has critical role in binding and entry of porcine epidemic diarrhea virus. Virus Res 227:150-157

10. Lee C (2015) Porcine epidemic diarrhea virus: an emerging and re-emerging epizootic swine virus. Virol J 12:193

11. Lee S, Son KY, Noh YH, Lee SC, Choi HW, Yoon IJ, Lee C (2017) Genetic characteristics, pathogenicity, and immunogenicity associated with cell adaptation of a virulent genotype $2 \mathrm{~b}$ porcine epidemic diarrhea virus. Vet Microbiol 207:248-258

12. Li BX, Ge JW, Li YJ (2007) Porcine aminopeptidase $\mathrm{N}$ is a functional receptor for the PEDV coronavirus. Virology 365:166-172

13. Li C, Li W, Lucio de Esesarte E, Guo H, van den Elzen P, Aarts E, van den Born E, Rottier PJM, Bosch BJ (2017) Cell attachment domains of the porcine epidemic diarrhea virus spike protein are key targets of neutralizing antibodies. J Virol. https://doi. org/10.1128/JVI.00273-17

14. Li F (2016) Structure, function, and evolution of coronavirus spike proteins. Annu Rev Virol 3:237-261

15. Li W, van Kuppeveld FJM, He Q, Rottier PJM, Bosch BJ (2016) Cellular entry of the porcine epidemic diarrhea virus. Virus Res 226:117-127

16. Li W, Hulswit RJG, Widjaja I, Raj VS, McBride R, Peng W, Widagdo W, Tortorici MA, van Dieren B, Lang Y, van Lent JWM, Paulson JC, de Haan CAM, de Groot RJ, van Kuppeveld FJM, Haagmans BL, Bosch BJ (2017) Identification of sialic acid-binding function for the Middle East respiratory syndrome coronavirus spike glycoprotein. Proc Natl Acad Sci USA 114:E8508-E8517

17. Li W, Luo R, He Q, van Kuppeveld FJM, Rottier PJM, Bosch BJ (2017) Aminopeptidase $\mathrm{N}$ is not required for porcine epidemic diarrhea virus cell entry. Virus Res 235:6-13 
18. Lin CM, Hou Y, Marthaler DG, Gao X, Liu X, Zheng L, Saif LJ, Wang Q (2017) Attenuation of an original US porcine epidemic diarrhea virus strain PC22A via serial cell culture passage. Vet Microbiol 201:62-71

19. Liu C, Tang J, Ma Y, Liang X, Yang Y, Peng G, Qi Q, Jiang S, Li J, Du L, Li F (2015) Receptor usage and cell entry of porcine epidemic diarrhea coronavirus. J Virol 89:6121-6125

20. Liu C, Ma Y, Yang Y, Zheng Y, Shang J, Zhou Y, Jiang S, Du L, Li J, Li F (2016) Cell entry of porcine epidemic diarrhea coronavirus is activated by lysosomal proteases. J Biol Chem 291:24779-24786

21. Matsuyama S, Ujike M, Morikawa S, Tashiro M, Taguchi F (2005) Protease-mediated enhancement of severe acute respiratory syndrome coronavirus infection. Proc Natl Acad Sci USA 102:12543-12547

22. Nam E, Lee C (2010) Contribution of the porcine aminopeptidase $\mathrm{N}$ (CD13) receptor density to porcine epidemic diarrhea virus infection. Vet Microbiol 144:41-50

23. Nogales A, Baker SF, Domm W, Martinez-Sobrido L (2016) Development and applications of single-cycle infectious influenza A virus (sciIAV). Virus Res 216:26-40

24. Oh JS, Song DS, Park BK (2003) Identification of a putative cellular receptor $150 \mathrm{kDa}$ polypeptide for porcine epidemic diarrhea virus in porcine enterocytes. J Vet Sci 4:269-275

25. Oka T, Saif LJ, Marthaler D, Esseili MA, Meulia T, Lin CM, Vlasova AN, Jung K, Zhang Y, Wang Q (2014) Cell culture isolation and sequence analysis of genetically diverse US porcine epidemic diarrhea virus strains including a novel strain with a large deletion in the spike gene. Vet Microbiol 173:258-269

26. Park JE, Cruz DJ, Shin HJ (2014) Clathrin- and serine proteasesdependent uptake of porcine epidemic diarrhea virus into Vero cells. Virus Res 191:21-29

27. Park JE, Li K, Barlan A, Fehr AR, Perlman S, McCray PB Jr, Gallagher T (2016) Proteolytic processing of Middle East respiratory syndrome coronavirus spikes expands virus tropism. Proc Natl Acad Sci USA 113:12262-12267

28. Schultze B, Krempl C, Ballesteros ML, Shaw L, Schauer R, Enjuanes L, Herrler G (1996) Transmissible gastroenteritis coronavirus, but not the related porcine respiratory coronavirus, has a sialic acid ( $N$-glycolylneuraminic acid) binding activity. J Virol 70:5634-5637

29. Shirato K, Maejima M, Islam MT, Miyazaki A, Kawase M, Matsuyama S, Taguchi F (2016) Porcine aminopeptidase N is not a cellular receptor of porcine epidemic diarrhea virus, but promotes its infectivity via aminopeptidase activity. J Gen Virol 97:2528-2539

30. Simmons G, Gosalia DN, Rennekamp AJ, Reeves JD, Diamond SL, Bates P (2005) Inhibitors of cathepsin L prevent severe acute respiratory syndrome coronavirus entry. Proc Natl Acad Sci USA 102:11876-11881

31. Sun M, Ma J, Yu Z, Pan Z, Lu C, Yao H (2017) Identification of two mutation sites in spike and envelope proteins mediating optimal cellular infection of porcine epidemic diarrhea virus from different pathways. Vet Res 48:44

32. Wang H, Yang P, Liu K, Guo F, Zhang Y, Zhang G, Jiang C (2008) SARS coronavirus entry into host cells through a novel clathrin- and caveolae-independent endocytic pathway. Cell Res 18:290-301

33. Wang J, Deng F, Ye G, Dong W, Zheng A, He Q, Peng G (2016) Comparison of lentiviruses pseudotyped with $\mathrm{S}$ proteins from coronaviruses and cell tropisms of porcine coronaviruses. Virol Sin 31:49-56

34. Wanitchang A, Jengarn J, Jongkaewwattana A (2011) The N terminus of PA polymerase of swine-origin influenza virus H1N1 determines its compatibility with PB2 and PB1 subunits through a strain-specific amino acid serine 186. Virus Res 155:325-333

35. Wanitchang A, Narkpuk J, Jaru-ampornpan P, Jengarn J, Jongkaewwattana A (2012) Inhibition of influenza A virus replication by influenza $B$ virus nucleoprotein: an insight into interference between influenza A and B viruses. Virology 432:194-203

36. Wanitchang A, Wongthida P, Jongkaewwattana A (2016) Influenza B virus M2 protein can functionally replace its influenza A virus counterpart in promoting virus replication. Virology 498:99-108

37. Wongthida P, Liwnaree B, Wanasen N, Narkpuk J, Jongkaewwattana A (2017) The role of ORF3 accessory protein in replication of cell-adapted porcine epidemic diarrhea virus (PEDV). Arch Virol 162:2553-2563 